\title{
Application Of Naive Bayes Algorithm In Classification Of Child Nutrition At The Simalungun Health Office
}

\author{
Susi Septi Hardiani ${ }^{1}$, M. Safii ${ }^{1}$, Dedi Suhendro ${ }^{2}$ \\ ${ }^{1}$ Informatics Engineering Study Program, STIKOM Tunas Bangsa, Pematangsiantar \\ ${ }^{2}$ Computerized Accounting Study Program, Amik Tunas Bangsa, Indonesia
}

\section{Article Info}

Article history:

Received Sep 9, 2021

Revised Sep 20, 2021

Accepted Oct 09, 2021

\section{Keywords:}

Data Mining;

Child Nutrition;

Nave Bayes;

Classification.

\begin{abstract}
Toddlers are among the most vulnerable groups to nutritional problems when viewed from the point of view of health and nutrition problems, while at this time they are experiencing a cycle of relatively rapid growth and development $7 \%$ is quite high where the number of births is relatively large. Researchers try to classify to toddlers using WEKA to find out whether they have nutritional disorders or are normal by using 5 attributes as system input and a class namely nutrition which divides this class into 4 namely bad, less, good and more with the amount of training data 219 data then data compared with the actual nutritional conditions and obtained an accuracy of $60 \%$ and an error of $40 \%$ with these results it can be concluded that the accuracy is not too good. Based on this, it is hoped that the results of this classification can help further research in classifying the nutrition of children under five.
\end{abstract}

This is an open access article under the CC BY-NC license.

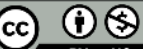

\section{Corresponding Author:}

\section{Safii}

Informatics Engineering Study Program,

STIKOM Tunas Bangsa,

Jl. Jend. Sudirman Blok A No.1-2 Pematangsiantar, North Sumatera-Indonesia

Email:m.safii@amiktunasbangsa.ac.id

\section{INTRODUCTION}

Children are an investment and hope for the future of the nation as well as the next generation in the future. In the life cycle, childhood is a phase where children experience growth and development that determines their future. It is necessary to optimize the development of children, because besides being crucial at that time children need attention and affection from parents or families so that fundamentally the rights and needs of children can be fulfilled properly[1]. Children must be able to grow and develop into human beings who are physically and mentally healthy, intelligent, happy, of high moral character and commendable, because in the future they are assets that will determine the quality of the nation's civilization[2]. At that age the child is in a period of human growth and development called the golden age. Based on several studies, it is stated that the golden age is present in the period of conception, namely since humans are still in the mother's womb until the first few years of their birth which is termed early age[3]. After the child is 24 months old, there is no 
more addition of new neuron cells as happened at the previous age, but maturation still continues until the child is four or five years old[4].

In the early stages of life, starting at the age of 3 years, children begin to be able to accept skills as the basis for forming knowledge and thinking processes. The role of parenting includes care and education, providing adequate food for the development of intellectual intelligence, nonmaterial provision for the development of emotional and spiritual intelligence greatly determines the quality of children in the future[5]. According to UNICEF, children's nutritional status is directly affected by food intake and infectious diseases[6]. Malnutrition is the basic cause of children's growth disorders, therefore, it must be prevented so that growth disorders do not occur, although children's physical growth disorders can still be corrected in the future by increasing good nutritional intake, but not so with the development of intelligence[7] .

In computer science there are many branches of science and methods contained therein such as Decision Support Systems, Expert Systems, Data Mining and others. Data mining is a process that employs one or more computer learning techniques (machine learning) to analyze and extract knowledge automatically[8],[9],[10]. There are 5 roles of data mining, namely estimation, prediction, classification, clustering, association[11],[12]. There are many algorithms in classification data mining, one of which is Nave Bayes. Naive Bayes algorithm is one of the algorithms contained in the classification technique[13],[14]. Naive Bayes is a classification with probability and statistical methods proposed by British scientist Thomas Bayes, which predicts future opportunities based on previous experience, so it is known as Bayes' theorem[15],[16]. The theorem is combined with Naive where it is assumed that the conditions between attributes are independent[17]. Naive Bayes classification assumes that the presence or absence of certain characteristics of a class has nothing to do with the characteristics of other classes.

\section{RESEARCH METHOD}

The research method is a technique or seeking, obtaining, collecting data, both in the form of primary data and secondary data used for the purpose of compiling a scientific work and analyzing factors related to the main issues so that there will be a truth of the data to be obtained. obtained. The settlement method used in this study is the data mining method with the Naive Bayes algorithm[18]. This research was conducted to implement the Naive Bayes algorithm in the classification of children's nutrition at the Simalungun Health Office. From the results of the classification of Child Nutrition data, the Simalungun Health Office can find out information so that it can be handled with Child Nutrition. In conducting research to obtain data, the method used is the Observation method, namely seeing and studying problems that exist in the field related to the object under study and the Literature Review method to find materials that support the definition of problems through books, papers, the internet, which closely related to the object of the problem[19].

The flow diagram of the research work activities carried out in this study is shown in Figure below: 


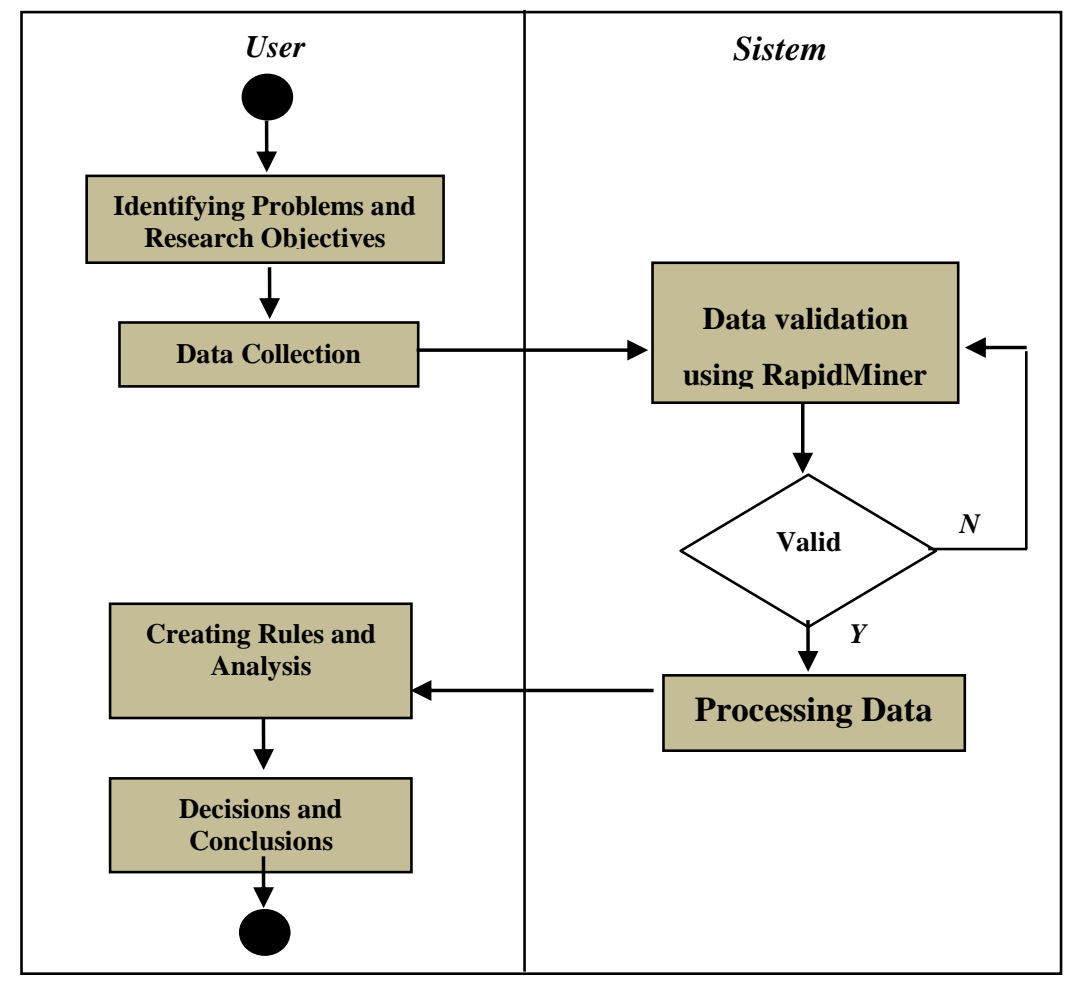

Figure 1. Research Activity Work Diagram

The data to be processed is 50 samples of child data in the Simalungun district health office as training data and testing data taken from the last 15 data from training data.

Table 1. Testing Data

\begin{tabular}{rcccccc}
\hline No & Sample & Gender & Age & Weight & Height & Status \\
\hline 1 & A36 & Boy & Age $>12$ and $<=24$ & Very thin & Short & Good Nutrition \\
2 & A37 & Boy & Age $>12$ and $<=24$ & Thin & Short & Malnutrition \\
3 & A38 & Boy & Age $>12$ and $<=24$ & Very thin & Very Short & Good Nutrition \\
4 & A39 & Boy & Age $<=12$ & Very thin & Very Short & Malnutrition \\
5 & A40 & Girl & Age $>12$ and $<=24$ & Very thin & Very Short & Malnutrition \\
6 & A41 & Girl & Age $<=12$ & Very thin & Normal & Good Nutrition \\
7 & A42 & Boy & Age $<=12$ & Very thin & Normal & Good Nutrition \\
8 & A43 & Boy & Age $>12$ and $<=24$ & Normal & Short & Good Nutrition \\
9 & A44 & Boy & Age $<=12$ & Thin & Short & Malnutrition \\
10 & A45 & Boy & Age $>12$ and $<=24$ & Normal & Short & Good Nutrition \\
11 & A46 & Boy & Age $>12$ and $<=24$ & Thin & Short & Malnutrition \\
12 & A47 & Girl & Age $>12$ and $<=24$ & Thin & Short & Malnutrition \\
13 & A48 & Girl & Age $>12$ and $<=24$ & Normal & Short & Good Nutrition \\
14 & A49 & Boy & Age $>12$ and $<=24$ & Very thin & Very Short & Malnutrition \\
15 & A50 & Boy & Age $>12$ and $<=24$ & Thin & Normal & Good Nutrition \\
\hline
\end{tabular}

3. RESULTS AND DISCUSSIONS 
To solve the problem in this research, data mining techniques with the Naïve Bayes algorithm are used as follows[20] :

$$
P(H \backslash X)=\frac{P(H \backslash X) *(P(H)}{P(X)}
$$

Where:

X : Data with unknown class

$\mathrm{H} \quad$ : Hypothesis data is a specific class

$\mathrm{P}(\mathrm{H} \mid \mathrm{X})$ : Probability of hypothesis $\mathrm{H}$ based on condition $\mathrm{X}$ (posteriori probability)

$\mathrm{P}(\mathrm{H}) \quad$ : Hypothesis probability $\mathrm{H}$ (prior probability)

$\mathrm{P}(\mathrm{X} \mid \mathrm{H})$ : Probability of $\mathrm{X}$ based on the conditions on the hypothesis $\mathrm{H}$

$\mathrm{P}(\mathrm{X}) \quad$ : Probability $\mathrm{X}$

\section{1. $\quad$ Solution with Nä̈ve Bayes}

The amount of data used is 50 data, Good Nutrition data is 27 and Malnutrition data is 23 data. In step 1, an indication of Nutritional Status has been obtained which is predicted based on Gender, Age, Body Weight and Body Height, according to the decision table 2.

Tabel 2. Data Training

\begin{tabular}{|c|c|c|c|c|c|c|}
\hline No & Sample & Gender & Age (o-24) & Weight & Height & Status \\
\hline 1 & $\mathrm{~A}_{1}$ & Boy & Age $<=12$ & Thin & Normal & Malnutrition \\
\hline 2 & $\mathrm{~A} 2$ & Girl & Age $>12$ and $<=24$ & Fat & Short & Good Nutrition \\
\hline 3 & $\mathrm{~A}_{3}$ & Boy & Age $>12$ and $<=24$ & Thin & Very Short & Malnutrition \\
\hline 4 & $\mathrm{~A}_{4}$ & Boy & Age $>12$ and $<=24$ & Very thin & Normal & Good Nutrition \\
\hline 5 & $\mathrm{~A}_{5}$ & Boy & Age $>12$ and $<=24$ & Thin & Normal & Good Nutrition \\
\hline 6 & A6 & Girl & Age $>12$ and $<=24$ & Very thin & Very Short & Malnutrition \\
\hline 7 & $\mathrm{~A}_{7}$ & Girl & Age $>12$ and $<=24$ & Normal & Short & Malnutrition \\
\hline 8 & A8 & Girl & Age $<=12$ & Fat & Very Short & Malnutrition \\
\hline 9 & A9 & Boy & Age $<=12$ & Very thin & Very Short & Malnutrition \\
\hline 10 & Aio & Boy & Age $<=12$ & Very thin & Normal & Good Nutrition \\
\hline 11 & An1 & Boy & Age $>12$ and $<=24$ & Very thin & Very Short & Malnutrition \\
\hline 12 & $A_{12}$ & Boy & Age $<=12$ & Normal & Normal & Good Nutrition \\
\hline 13 & $\mathrm{~A}_{13}$ & Girl & Age $<=12$ & Very thin & Normal & Malnutrition \\
\hline 14 & $\mathrm{~A} 14$ & Girl & Age $>12$ and $<=24$ & Normal & Short & Good Nutrition \\
\hline 15 & $\mathrm{~A}_{15}$ & Girl & Age $>12$ and $<=24$ & Fat & Normal & Good Nutrition \\
\hline 16 & A 6 & Boy & Age $>12$ and $<=24$ & Normal & Short & Good Nutrition \\
\hline 17 & $\mathrm{~A}_{17}$ & Boy & Age $<=12$ & Normal & Normal & Good Nutrition \\
\hline 18 & $\mathrm{~A}_{1} 8$ & Boy & Age $<=12$ & Very thin & Short & Malnutrition \\
\hline 19 & A19 & Boy & Age $>12$ and $<=24$ & Very thin & Short & Malnutrition \\
\hline 20 & $\mathrm{~A} 2 \mathrm{O}$ & Boy & Age $>12$ and $<=24$ & Thin & Short & Malnutrition \\
\hline 21 & A21 & Boy & Age $<=12$ & Thin & Short & Malnutrition \\
\hline 22 & $\mathrm{~A} 22$ & Boy & Age $>12$ and $<=24$ & Normal & Short & Good Nutrition \\
\hline 23 & $\mathrm{~A} 23$ & Boy & Age $<=12$ & Normal & Normal & Good Nutrition \\
\hline 24 & $\mathrm{~A} 24$ & Girl & Age $<=12$ & Thin & Normal & Good Nutrition \\
\hline 25 & A 25 & Girl & Age $>12$ and $<=24$ & Fat & Normal & Good Nutrition \\
\hline 26 & $\mathrm{~A} 26$ & Boy & Age $>12$ and $<=24$ & Normal & Short & Good Nutrition \\
\hline 27 & $\mathrm{~A} 27$ & Boy & Age $>12$ and $<=24$ & Thin & Very Short & Malnutrition \\
\hline 28 & $\mathrm{~A} 28$ & Boy & Age $>12$ and $<=24$ & Normal & Normal & Good Nutrition \\
\hline 29 & A29 & Girl & Age $>12$ and $<=24$ & Fat & Normal & Good Nutrition \\
\hline 30 & $\mathrm{~A}_{30}$ & Boy & Age $>12$ and $<=24$ & Normal & Short & Good Nutrition \\
\hline 31 & A31 & Boy & Age $>12$ and $<=24$ & Normal & Short & Good Nutrition \\
\hline 32 & $\mathrm{~A}_{32}$ & Boy & Age $>12$ and $<=24$ & Very thin & Very Short & Malnutrition \\
\hline 33 & A33 & Girl & Age $<=12$ & Very thin & Very Short & Malnutrition \\
\hline 34 & A34 & Girl & Age $<=12$ & Very thin & Short & Malnutrition \\
\hline
\end{tabular}




\begin{tabular}{|c|c|c|c|c|c|c|}
\hline No & Sample & Gender & Age (o-24) & Weight & Height & Status \\
\hline 35 & $A_{35}$ & Boy & Age $<=12$ & Fat & Normal & Good Nutrition \\
\hline 36 & A36 & Boy & Age $>12$ and $<=24$ & Very thin & Short & Good Nutrition \\
\hline 37 & $\mathrm{~A} 37$ & Boy & Age $>12$ and $<=24$ & Thin & Short & Malnutrition \\
\hline 38 & $\mathrm{~A} 38$ & Boy & Age $>12$ and $<=24$ & Very thin & Very Short & Good Nutrition \\
\hline 39 & А 39 & Boy & Age $<=12$ & Very thin & Very Short & Malnutrition \\
\hline 40 & $\mathrm{~A}_{40}$ & Girl & Age $>12$ and $<=24$ & Very thin & Very Short & Malnutrition \\
\hline 41 & $\mathrm{~A}_{41}$ & Girl & Age $<=12$ & Very thin & Normal & Good Nutrition \\
\hline 42 & $\mathrm{~A} 42$ & Boy & Age $<=12$ & Very thin & Normal & Good Nutrition \\
\hline 43 & $\mathrm{~A}_{43}$ & Boy & Age $>12$ and $<=24$ & Normal & Short & Good Nutrition \\
\hline 44 & A 44 & Boy & Age $<=12$ & Thin & Short & Malnutrition \\
\hline 45 & $\mathrm{~A} 45$ & Boy & Age $>12$ and $<=24$ & Normal & Short & Good Nutrition \\
\hline 46 & $\mathrm{~A}_{4} 6$ & Boy & Age $>12$ and $<=24$ & Thin & Short & Malnutrition \\
\hline 47 & $\mathrm{~A} 47$ & Girl & Age $>12$ and $<=24$ & Thin & Short & Malnutrition \\
\hline 48 & $\mathrm{~A} 48$ & Girl & Age $>12$ and $<=24$ & Normal & Short & Good Nutrition \\
\hline 49 & A 49 & Boy & Age $>12$ and $<=24$ & Very thin & Very Short & Malnutrition \\
\hline 50 & A5o & Boy & Age $>12$ and $<=24$ & Thin & Normal & Good Nutrition \\
\hline
\end{tabular}

a. Counting the Number of Classes/Labels

Based on the training data above, it can be calculated by the following equation formula:

Status Good Nutrition Status $=\frac{\text { Number of Possible Good Nutrition }}{\text { Number of Possible Nutritional Status }}=\frac{27}{50}=0,54$ or $54 \%$

Malnutrition Status $=\frac{\text { Number of Possible Malnutrition }}{\text { Number of Possible Nutritional Status }}=\frac{23}{50}=0,46$ or $46 \%$

b. Counting the number of cases with the same class

After looking for the probability value of each criterion, obtained from the training data, the probability value for each criterion can be seen in the following table 2 With the following equation formula:

P. Good Nutrition, boys $=\frac{\text { Same Number of Cases }}{\text { Total Cases }(\text { class }) \text { are the same }}=\frac{19}{27}=0,70$

P. Good Nutrition, boys $=\frac{\text { Same Number of Cases }}{\text { Total Cases }(\text { class }) \text { are the same }}=\frac{8}{27}=0,30$

P. Malnutrition, Girls $=\frac{\text { Jumlah Kasus Yang sama }}{\text { Total Cases }(\text { class }) \text { are the same }}=\frac{15}{23}=0,65$

P. Malnutrition, Girls $=\frac{\text { Same Number of Cases }}{\text { Total Cases }(\text { class }) \text { are the same }}=\frac{8}{23}=0,35$

Table 3. Table of Gender Probability Values

\begin{tabular}{lcccc}
\hline \multirow{2}{*}{ P=Gender } & \multicolumn{2}{c}{ Nutrition Status } & \multicolumn{2}{c}{ Probilitas } \\
& Good Nutrition & Malnutrition & Good Nutrition & Malnutrition \\
\hline Boy & 19 & 15 & 0,70 & 0,65 \\
Girl & 8 & 8 & 0,30 & 0,35 \\
Total & 27 & 23 & 1 & 1 \\
\hline
\end{tabular}

Table 4. Age Probability Value Table

\begin{tabular}{lcccc}
\hline \multirow{2}{*}{$\mathrm{P}=$ Age $(\mathrm{o}-24)$} & \multicolumn{2}{c}{ Nutrition Status } & \multicolumn{2}{c}{ Probilitas } \\
& Good Nutrition & Malnutrition & Good Nutrition & Malnutrition \\
\hline Age $<=12$ & 8 & 10 & 0,30 & 0,43 \\
Age $>12$ and $<=24$ & 19 & 13 & 0,70 & 0,57 \\
\hline
\end{tabular}




\begin{tabular}{lllll}
\hline Total & 27 & 23 & 1 & 1 \\
\hline
\end{tabular}

Table 5. Table of Probability Values for Body Weight

\begin{tabular}{lcccc}
\hline \multirow{2}{*}{ P=Weight } & \multicolumn{2}{c}{ Nutrition Status } & \multicolumn{2}{c}{ Probility } \\
& Good Nutrition & Malnutrition & Good Nutrition & Malnutrition \\
\hline Fat & 5 & 1 & 0,19 & 0,04 \\
Normal & 13 & 1 & 0,48 & 0,04 \\
Thin & 3 & 9 & 0,11 & 0,39 \\
Very thin & 6 & 12 & 0,22 & 0,52 \\
Total & 27 & 23 & 1 & 1 \\
\hline
\end{tabular}

Tabel 6. Tabel Nilai Probabilitas Tinggi Baand

\begin{tabular}{lcccc}
\hline \multirow{2}{*}{ P=Height } & \multicolumn{2}{c}{ Nutrition Status } & \multicolumn{2}{c}{ Probility } \\
& Good Nutrition & Malnutrition & Good Nutrition & Malnutrition \\
\hline Normal & 15 & 2 & 0,56 & 0,09 \\
Short & 11 & 10 & 0,41 & 0,43 \\
Very Short & 1 & 11 & 0,04 & 0,48 \\
Total & 27 & 23 & 1 & 1 \\
\hline
\end{tabular}

c. Multiply All Results Variable Good Nutrition and Malnutrition

The next step is to multiply all the results of the Good Nutrition and Malnutrition variables using the test data. The following is the test data used in the following table 7 .

\begin{tabular}{rcccccc}
\hline \multicolumn{7}{c}{ Table 7. Test Data } \\
\hline No & Sample & Gender & Age $(0-24)$ & Weight & Height & Status \\
\hline 1 & A36 & Boy & Age $>12$ and $<=24$ & Very thin & Short & $?$ \\
2 & A37 & Boy & Age $>12$ and $<=24$ & Thin & Short & $?$ \\
3 & A38 & Boy & Age $>12$ and $<=24$ & Very thin & Very Short & $?$ \\
4 & A39 & Boy & Age $<=12$ & Very thin & Very Short & $?$ \\
5 & A40 & Girl & Age $>12$ and $<=24$ & Very thin & Very Short & $?$ \\
6 & A41 & Girl & Age $<=12$ & Very thin & Normal & $?$ \\
7 & A42 & Boy & Age $<=12$ & Very thin & Normal & $?$ \\
8 & A43 & Boy & Age $>12$ and $<=24$ & Normal & Short & $?$ \\
9 & A44 & Boy & Age $<=12$ & Thin & Short & $?$ \\
10 & A45 & Boy & Age $>12$ and $<=24$ & Normal & Short & $?$ \\
11 & A46 & Boy & Age $>12$ and $<=24$ & Thin & Short & $?$ \\
12 & A47 & Girl & Age $>12$ and $<=24$ & Thin & Short & $?$ \\
13 & A48 & Girl & Age $>12$ and $<=24$ & Normal & Short & $?$ \\
14 & A49 & Boy & Age $>12$ and $<=24$ & Very thin & Very Short & $?$ \\
15 & A50 & Boy & Age $>12$ and $<=24$ & Thin & Normal & $?$ \\
\hline
\end{tabular}

The classification results are not yet known because these variables are predicted results from data calculated from each variable. Here is the calculation process:

Sample $($ Status $)=$ Gender * Age $(0-24) *$ Weight *Hight

A36 $($ Good Nutrition $)=$ Boy * Age $>12$ and $<=24$ * Very thin * Short

$$
\begin{aligned}
& =0,70 * 0,70 * 0,22 * 0,41 \\
& =0,024
\end{aligned}
$$

A36 (Malnutrition) $=$ Boy * Age $>12$ and $<=24$ * Very thin * Short

$$
\begin{aligned}
& =0,65 * 0,57 * 0,52 * 0,43 \\
& =0,038
\end{aligned}
$$


A 37 (Good Nutrition $)=$ Boy * Age $>12$ and $<=24$ * Thin * Short

$$
\begin{aligned}
& =0,70 * 0,70 * 0,11 * 0,41 \\
& =0,012
\end{aligned}
$$

A 37 (Malnutrition) $=$ Boy * Age $>12$ and $<=24$ * Thin * Short

$$
\begin{aligned}
& =0,65 * 0,57 * 0,39 * 0,43 \\
& =0,029
\end{aligned}
$$

A38 $($ Good Nutrition $)=0,002$

A38 (Malnutrition) $=0,042$

A39 $($ Good Nutrition $)=0,001$

A39 (Malnutrition) $=0,033$

A40 (Good Nutrition) $=0,001$

A 40 (Malnutrition $)=0,023$

\begin{tabular}{|c|c|c|c|c|c|c|c|}
\hline No & Sample & Gender & Age (o - 24) & Height & Hight & Good Nutrition & Malnutrition \\
\hline 1 & $\mathrm{~A}_{3} 6$ & Boy & Age $>12$ and $<=24$ & Very thin & Short & 0,024 & 0,038 \\
\hline 2 & A37 & Boy & Age $>12$ and $<=24$ & Thin & Short & 0,012 & 0,029 \\
\hline 3 & $\mathrm{~A}_{3} 8$ & Boy & Age $>12$ and $<=24$ & Very thin & Very Short & 0,002 & 0,042 \\
\hline 4 & A39 & Boy & Age $<=12$ & Very thin & Very Short & 0,001 & 0,033 \\
\hline 5 & $\mathrm{~A}_{40}$ & Girl & Age $>12$ and $<=24$ & Very thin & Very Short & 0,001 & 0,023 \\
\hline 6 & $\mathrm{~A}_{41}$ & Girl & Age $<=12$ & Very thin & Normal & 0,006 & 0,003 \\
\hline 7 & $\mathrm{~A}_{42}$ & Boy & Age $<=12$ & Very thin & Normal & 0,014 & 0,006 \\
\hline 8 & $\mathrm{~A}_{43}$ & Boy & Age $>12$ and $<=24$ & Normal & Short & 0,052 & 0,003 \\
\hline 9 & $\mathrm{~A}_{44}$ & Boy & Age $<=12$ & Thin & Short & 0,005 & 0,022 \\
\hline 10 & $\mathrm{~A}_{45}$ & Boy & Age $>12$ and $<=24$ & Normal & Short & 0,052 & 0,003 \\
\hline 11 & $\mathrm{~A} 46$ & Boy & Age $>12$ and $<=24$ & Thin & Short & 0,012 & 0,029 \\
\hline 12 & $\mathrm{~A} 47$ & Girl & Age $>12$ and $<=24$ & Thin & Short & 0,005 & 0,015 \\
\hline 13 & $\mathrm{~A}_{4} 8$ & Girl & Age $>12$ and $<=24$ & Normal & Short & 0,022 & 0,002 \\
\hline 14 & A49 & Boy & Age $>12$ and $<=24$ & Very thin & Very Short & 0,002 & 0,042 \\
\hline 15 & A5o & Boy & Age $>12$ and $<=24$ & Thin & Normal & 0,017 & 0,006 \\
\hline
\end{tabular}

A50 (Good Nutrition) $=0,017$

A5o (Malnutrition) $=0,006$

Table 8. Calculation Results of Each Variable

d. Compare Good Nutrition and Malnutrition Class Results

From the results of these calculations, compare the probability values between class $(\mathrm{P} \mid \mathrm{Good}$ Nutrition) and class ( $\mathrm{P} \mid$ Malnutrition). The highest probability value can be seen in the following table 9: 
Table 9. Comparison Results

\begin{tabular}{|c|c|c|c|c|c|c|c|c|c|}
\hline $\begin{array}{l}\text { Sampl } \\
\text { e }\end{array}$ & $\begin{array}{l}\text { Gende } \\
\mathbf{r}\end{array}$ & Age (o - 24) & Weight & Height & $\begin{array}{l}\text { Nutrition } \\
\text { Status }\end{array}$ & & $\begin{array}{c}\text { Class } \\
\text { Predictio } \\
\text { n }\end{array}$ & $\begin{array}{c}\text { Good } \\
\text { Nutritio } \\
\text { n }\end{array}$ & $\begin{array}{c}\text { Malnutriti } \\
\text { on }\end{array}$ \\
\hline $\mathrm{A}_{3} 6$ & Boy & $\begin{array}{c}\text { Age }>12 \text { and } \\
<=24\end{array}$ & $\begin{array}{l}\text { Very } \\
\text { thin }\end{array}$ & Short & $\begin{array}{c}\text { Good } \\
\text { Nutrition }\end{array}$ & & $\begin{array}{l}\text { Malnutriti } \\
\text { on }\end{array}$ & 0,024 & 0,038 \\
\hline $\mathrm{A}_{37}$ & Boy & $\begin{array}{c}\text { Age }>12 \text { and } \\
<=24\end{array}$ & Thin & Short & Malnutrition & & $\begin{array}{l}\text { Malnutriti } \\
\text { on }\end{array}$ & 0,012 & 0,029 \\
\hline $\mathrm{A} 38$ & Boy & $\begin{array}{c}\text { Age }>12 \text { and } \\
<=24\end{array}$ & $\begin{array}{l}\text { Very } \\
\text { thin }\end{array}$ & $\begin{array}{l}\text { Very } \\
\text { Short }\end{array}$ & $\begin{array}{c}\text { Good } \\
\text { Nutrition }\end{array}$ & & $\begin{array}{l}\text { Malnutriti } \\
\text { on }\end{array}$ & 0,002 & 0,042 \\
\hline A39 & Boy & Age <=12 & $\begin{array}{l}\text { Very } \\
\text { thin }\end{array}$ & $\begin{array}{l}\text { Very } \\
\text { Short }\end{array}$ & Malnutrition & & $\begin{array}{l}\text { Malnutriti } \\
\text { on }\end{array}$ & 0,001 & 0,033 \\
\hline $\mathrm{A}_{40}$ & Girl & $\begin{array}{c}\text { Age }>12 \text { and } \\
<=24\end{array}$ & $\begin{array}{l}\text { Very } \\
\text { thin }\end{array}$ & $\begin{array}{l}\text { Very } \\
\text { Short }\end{array}$ & Malnutrition & & $\begin{array}{l}\text { Malnutriti } \\
\text { on }\end{array}$ & 0,001 & 0,023 \\
\hline $\mathrm{A}_{41}$ & Girl & Age $<=12$ & $\begin{array}{l}\text { Very } \\
\text { thin }\end{array}$ & Normal & $\begin{array}{c}\text { Good } \\
\text { Nutrition }\end{array}$ & & $\begin{array}{c}\text { Good } \\
\text { Nutrition }\end{array}$ & o,oo6 & 0,003 \\
\hline $\mathrm{A}_{42}$ & Boy & Age $<=12$ & $\begin{array}{l}\text { Very } \\
\text { thin }\end{array}$ & Normal & $\begin{array}{c}\text { Good } \\
\text { Nutrition }\end{array}$ & . & $\begin{array}{c}\text { Good } \\
\text { Nutrition }\end{array}$ & 0,014 & 0,006 \\
\hline $\mathrm{A}_{43}$ & Boy & $\begin{array}{c}\text { Age }>12 \text { and } \\
<=24\end{array}$ & Normal & Short & $\begin{array}{c}\text { Good } \\
\text { Nutrition }\end{array}$ & 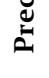 & $\begin{array}{c}\text { Good } \\
\text { Nutrition }\end{array}$ & 0,052 & 0,003 \\
\hline $\mathrm{A} 44$ & Boy & Age $<=12$ & Thin & Short & Malnutrition & & $\begin{array}{l}\text { Malnutriti } \\
\text { on }\end{array}$ & 0,005 & 0,022 \\
\hline $\mathrm{A}_{45}$ & Boy & $\begin{array}{c}\text { Age }>12 \text { and } \\
<=24\end{array}$ & Normal & Short & $\begin{array}{c}\text { Good } \\
\text { Nutrition }\end{array}$ & & $\begin{array}{c}\text { Good } \\
\text { Nutrition }\end{array}$ & 0,052 & 0,003 \\
\hline $\mathrm{A}_{4} 6$ & Boy & $\begin{array}{c}\text { Age }>12 \text { and } \\
<=24\end{array}$ & Thin & Short & Malnutrition & & $\begin{array}{l}\text { Malnutriti } \\
\text { on }\end{array}$ & 0,012 & 0,029 \\
\hline $\mathrm{A}_{47}$ & Girl & $\begin{array}{c}\text { Age }>12 \text { and } \\
<=24\end{array}$ & Thin & Short & Malnutrition & & $\begin{array}{l}\text { Malnutriti } \\
\text { on }\end{array}$ & 0,005 & 0,015 \\
\hline $\mathrm{A}_{4} 8$ & Girl & $\begin{array}{c}\text { Age }>12 \text { and } \\
<=24\end{array}$ & Normal & Short & $\begin{array}{c}\text { Good } \\
\text { Nutrition }\end{array}$ & & $\begin{array}{l}\text { Good } \\
\text { Nutrition }\end{array}$ & 0,022 & 0,002 \\
\hline A49 & Boy & $\begin{array}{c}\text { Age }>12 \text { and } \\
<=24\end{array}$ & $\begin{array}{l}\text { Very } \\
\text { thin }\end{array}$ & $\begin{array}{l}\text { Very } \\
\text { Short }\end{array}$ & Malnutrition & & $\begin{array}{l}\text { Malnutriti } \\
\text { on }\end{array}$ & 0,002 & 0,042 \\
\hline $\mathrm{A}_{50}$ & Boy & $\begin{array}{c}\text { Age }>12 \text { and } \\
<=24\end{array}$ & Thin & Normal & $\begin{array}{c}\text { Good } \\
\text { Nutrition }\end{array}$ & & $\begin{array}{c}\text { Good } \\
\text { Nutrition }\end{array}$ & 0,017 & 0,006 \\
\hline
\end{tabular}

Table 1o. Confusion Table

\begin{tabular}{|l|c|c|}
\cline { 2 - 3 } \multicolumn{1}{c|}{} & \multicolumn{2}{c|}{ Class } \\
\hline Prediction & Good Nutrition & Malnutrition \\
\hline Good Nutrition & 6 & 0 \\
\hline Malnutrition & 2 & 7 \\
\hline
\end{tabular}

Accuracy Count $=$ Prediction $($ Good Nutrition, Good Class $)+$ Prediction $($ Malnutrition, Poor Class)/ Total Number of Predictions (Good Nutrition, Good Class) and Prediction (Malnutrition, Poor Class)

$$
\begin{aligned}
& =(6+7) / 15 \\
& =0,87 \text { atau } 87 \%
\end{aligned}
$$

From the results of these calculations, comparing the probability value between the class (P|Good Nutrition) and the class ( $\mathrm{P} \mid$ Malnutrition) the highest probability value can be concluded that the Nutritional Status has an accuracy of $87 \%$.

\section{CONCLUSION}

Based on the results of the research conducted, it can be concluded that applying Data Mining using the Naïve Bayes algorithm on the classification of children's nutrition at the Simalungun Health Office can be applied properly. The source of data processing used in this study is data obtained directly at the Simalungun Health Office. The amount of data in this test is 15 samples of data tested 
from the comparison of Good Nutrition and Malnutrition. From the calculation results of the Naive Bayes Algorithm, the classification with Good Nutrition and Malnutrition is 6 Good Nutrition and 7 Malnutrition. Nutrition as many as 7 records. It can be concluded that the Simalungun Health Service provides good service to the community.

\section{ACKNOWLEDGEMENTS}

Thank you to the supervisors as lecturers at AMIK and STIKOM Tunas Bangsa Pematangsiantar who have assisted in the preparation of this research so that it has reached the publication stage. Thanks to all parties, especially Simalungun Health Office and it is hoped that this research can be used as a source of data that can assist in decision making. For the development and progress of this research title, we expect constructive input and criticism so that this research can be continued.

\section{REFERENCES}

[1] World Health Organization, "Early Childhood: Development and Disability," PsycCRITIQUES, vol. 33, no. 8, p. 269, 2012, [Online]. Available: http://content.apa.org/reviews/025945.

[2] A. L. Costa, "Teaching and Assessing Habits of Mind," vol. 96741, no. 808, 1999.

[3] R. H. Lestari, M. Mudhawaroh, and M. Ratnawati, "Intelligence Optimization in the Golden Age by Stimulating the Right-Brain in Mojokrapak Village, Tembelang District, Jombang Regency," Nucleus, vol. 1, no. 2, pp. 58-61, 2020, doi: 10.37010/nuc.vii2.166.

[4] A. Chapnick, “The golden age," Int. J., vol. 64, no. 1, pp. 205-221, 2008, doi: 10.1177/002070200906400118.

[5] M. Muslim, H. Ahmad, and S. Rahim, "The effect of emotional, spiritual and intellectual intelligence on auditor professionalism at the inspectorate of South Sulawesi Province," Indones. Account. Rev., vol. 9, no. 1, p. 73, 2019, doi: 10.14414/tiar.v9i1.1416.

[6] UNICEF., Children, food and nutrition : growing well in a changing world. 2019.

[7] I. G. Pratiwi and D. A. Restanty, "Pengaruh Metode Think, Pair and Share Terhadap Keterampilan Kader Dalam Pengisian Kms Balita,” JPP (Jurnal Kesehat. Poltekkes Palembang), vol. 13, no. 2, pp. 128-135, 2019, doi: 10.36o86/jpp.v13i2.237.

[8] W. Yulianti and Salmidi, "Metode Rough Set untuk Menganalisa Problematika Guru Dalam Menggunakan Media Pembelajaran Berbasis Komputer,” J. Teknol. dan Sist. Inf. Univrab, vol. 1, no. 1, pp. 19-25, 2016.

[9] Y. Cheng, K. Chen, H. Sun, Y. Zhang, and F. Tao, "Data and Knowledge Mining with Big Data towards Smart Production," J. Ind. Inf. Integr., vol. 9, Sep. 2017, doi: 10.1016/j.jii.2017.08.oo1.

[10] H. Tran, Survey of Machine Learning and Data Mining Techniques used in Multimedia System. 2019.

[11] R. Amanda and E. S. Negara, "Analysis and Implementation Machine Learning for YouTube Data Classification by Comparing the Performance of Classification Algorithms," J. Online Inform., vol. 5, no. 1, pp. 61-72, 2020, doi: 10.15575/join.v5i1.505.

[12] S. Suhada and E. Setiawan, "Classification Needs Teachers," no. Icisbc, pp. 408-414, 2013.

[13] A. W. Syaputri, E. Irwandi, and M. Mustakim, "Naïve Bayes Algorithm for Classification of Student Major's Specialization,” J. Intell. Comput. Heal. Informatics, vol. 1, no. 1, p. 17, 2020, doi: 10.26714/jichi.vii1.5570.

[14] I. B. A. Peling, I. N. Arnawan, I. P. A. Arthawan, and I. G. N. Janardana, "Implementation of Data Mining To Predict Period of Students Study Using Naive Bayes Algorithm,” Int. J. Eng. Emerg. Technol., vol. 2, no. 1, p. 53, 2017, doi: 10.24843/ijeet.2017.v02.io1.p11.

[15] M. J. Sodiq and E. I. Sela, "Perbandingan Metode Naive Bayes Dan K-Nearest Neighbor Pada Klasifikasi Kualitas Udara Di Dki Jakarta,” 2019.

[16] R. Rino, "The Comparison of Data Mining Methods Using C4.5 Algorithm and Naive Bayes in Predicting Heart Disease," Tech-E, vol. 4, no. 2, p. 44, 2021, doi: 10.31253/te.v4i2.543.

[17] Mustakim et al., "Data Sharing Technique Modeling for Naive Bayes Classifier for Eligibility Classification of Recipient Students in the Smart Indonesia Program,” J. Phys. Conf. Ser., vol. 1424, no. 1, 2019, doi: 10.1088/1742-6596/1424/1/012009.

[18] C. Fadlan, S. Ningsih, and A. Windarto, "PENERAPAN METODE NAÏVE BAYES DALAM KLASIFIKASI KELAYAKAN KELUARGA PENERIMA BERAS RASTRA,” J. Tek. Inform. Musirawas, vol. 3, p. 1, Jun. 2018, doi: 10.32767/jutim.v3i1.286.

[19] M. Collins, "Research methodology," J. Occup. Heal. Saf. - Aust. New Zeal., vol. 6, no. 5, p. 352, 1990.

[20] S. Jyothi and P. Bhargavi, "Applying Naive Bayes Data Mining Technique for Classification of 
Agricultural Land Soils," 57. P.Bhargavi, S. Jyothi, vol. 9, Aug. 2009.

[21] M. Tigor, "Bagaimana Menyatakan Permasalahan Riset (Research Problem/Problem Statement)?," Rudang Mayang Publ., vol. 1, no. 1, pp. 1-2, 2020 